\title{
THE
}

\section{Chaos in Spin Clusters: Correlation Functions and Spectral Properties}

\author{
Niraj Srivastava \\ Charles Kaufman \\ University of Rhode Island, ckaufman@uri.edu \\ Gerhard Müller \\ University of Rhode Island, gmuller@uri.edu
}

Follow this and additional works at: https://digitalcommons.uri.edu/phys_facpubs

Terms of Use

All rights reserved under copyright.

\section{Citation/Publisher Attribution}

Niraj Srivastava, Christoper Kaufman and Gerhard Müller. Chaos in spin clusters: correlation functions and spectral properties. J. Appl. Phys. 63 (1988), 4154-4156.

Available at: http://dx.doi.org/10.1063/1.340524

This Article is brought to you for free and open access by the Physics at DigitalCommons@URI. It has been accepted for inclusion in Physics Faculty Publications by an authorized administrator of DigitalCommons@URI. For more information, please contact digitalcommons-group@uri.edu. 


\section{Chaos in spin clusters: Correlation functions and spectral properties}

Niraj Srivastava, Charles Kauman, and Gerhard Muller

Department of Physics, The University of Rhode Island, Kingston, Rhode Island 028801-0817

We investigate dynamic correlation functions for a pair of exchange-coupled classical spins with biaxial exchange and/or single-site anisotropy. This represents a Hamiltonian system with two degrees of freedom for which we have previously established the integrability criteria. We discuss the impact of (non-) integrability on the autocorrelation functions and their spectral properties. We point out the role of long-time anomalies caused by low-flux cantori, which dominate the convergence properties of time averages and determine the long-time asymptotic behavior of autocorrelation functions in nonintegrable cases.

Classical spin dynamics of magnetic insulators, which employs classical counterparts of quantum mechanical exchange Hamiltonians, has proven to be very useful and illuminating in statistical mechanics and magnetic materials research. For a direct connection to experimentally observable quantities and for easy comparison with the results of quantum spin dynamics, it is convenient to focus such classical dynamical studies on the properties of dynamic correlation functions. Nonintegrability effects in spin dynamics have received their due share of attention only fairly recently. ${ }^{1-5}$ In fact, spin dynamics lends itself ideally to the study of classical and quantum chaos, which is necessarily numerical in its nature, for the following reasons: The "phase space" of a finite classical spin system is a compact manifold, and the energy is bounded by a finite interval; the Hibert space of a finite quantum spin system has a finite dimensionality. These special features allow for a numerical analysis with no further truncations or approximations which are likely to introduce artificial effects. The object of the work reported here is to study autocorrelation functions and their spectral properties in integrable and nonintegrable spin clusters.

Consider a system of two localized classical three-component spins $S_{l}=\left(S_{l s}^{x} S_{l}^{y}, S_{l}^{z}\right), l=1,2$, specified by an interaction Hamitonian of the general form

$$
H=\sum_{\alpha=x y z}\left\{-J_{\alpha} S_{1}^{\alpha} S_{2}^{\alpha}+\frac{1}{2} A_{\alpha}\left[\left(S_{\S}^{\alpha}\right)^{2}+\left(S_{2}^{\alpha}\right)^{2}\right]\right\}
$$

which includes both exchange and single-site anisotropy. It time evolution is governed by the equation of motion

$d \mathbf{S}_{l} / d t=-\mathbf{S}_{i} \times \partial H / \partial \mathbf{S}_{i}=\left\{H, \mathbf{S}_{i}\right\}, \quad l=1,2$,

where

$$
\left\{S_{!}^{a}, S_{l}^{\beta}\right\}=-\delta_{l,} \cdot \sum_{\gamma} \epsilon^{\alpha \beta \gamma} S \gamma
$$

are the Poisson brackets for classical spin variables. Their structure guarantees that the Hamilton equation of motion (2) is consistent with the Heisenberg equation of motion for quantum spin operators. If the classical spins $\mathbb{S}_{l}$ are $\mathrm{ex}$ pressed in terms of spherical coordinates as

$$
\mathrm{S}_{l}=S\left(\sin \vartheta_{l} \cos \varphi_{l}, \sin \vartheta_{l} \sin \varphi_{l}, \cos \vartheta_{l}\right)
$$

then a set of canonical variables is given by $p_{i}=S \cos \vartheta_{i}$ $q_{l}=\varphi_{l}$. Hence, the classical two-spin system (1) represents an autonomous Hamiltonian system of two degrees of freedom. It is completely integrable if there exists an independent integral of the motion in addition to $H$. In a previous study ${ }^{3}$ we have determined the following integrability condition for the two-spin model( 1$)$ :

$$
\begin{aligned}
& \left(A_{x}-A_{y}\right)\left(A_{y}-A_{z}\right)\left(A_{z}-A_{x}\right) \\
& \quad+\sum_{\alpha \beta \gamma=: c y c l(x y z)} J_{\alpha}^{2}\left(A_{\beta}-A_{\gamma}\right)=0
\end{aligned}
$$

and have constructed explicitly the second independent integral of the motion for the integrable cases.

In the present work, we study two types of autocorrelation functions for classical spin clusters:

(i) autocorrelation function as a time average,

$$
\begin{array}{r}
\left\langle S_{l}^{\alpha}(t) S_{l}^{\alpha}\right\rangle_{T}=\lim _{T \rightarrow \infty} \frac{1}{T} \int_{0}^{T} d t^{\prime} S_{l}^{\alpha}\left(t+t^{\prime}\right) S_{l}^{\alpha}\left(t^{\prime}\right), \\
l=1,2 ; \quad \alpha=x, y, z,
\end{array}
$$

where the quantity $S_{l}^{\alpha}(t) S_{l}^{\alpha}(0)$ is averaged over all points of a single phase-space trajectory. Since the phase flow generated by Hamiltonian (1) is not ergodic on the energy hypersurface even for nonintegrable cases, correlation functions defined as (microcanonical) phase averages are in general different from (5), but no less interesting in the context of our study. Here we focus our discussion of phase averages on canonical ensembles at infinite temperature:

(ii) autocorrelation function as a phase average,

$$
\begin{aligned}
& \left\langle S_{l}^{\alpha}(t) S_{l}^{\alpha}\right\rangle_{p} \\
& \quad=(4 \pi)^{-2} \int d \mathbf{S}_{1}(0) \int d \mathbf{S}_{2}(0) S_{l}^{\alpha}(t) S_{l}^{\alpha}(0),
\end{aligned}
$$

where the quantity $S_{l}^{\alpha}(t) S_{l}^{\alpha}(0)$ is determined as a function of the initial conditions $\left[S_{1}(0), S_{2}(0)\right]$ and then averaged over all initial conditions in phase space. Both types of autocorrelation functions are real and even functions of time.

In integrable cases of the two-spin model (1), the course of any trajectory is confined to an invariant torus, implying that the correlation function $\left\langle S_{l}^{\alpha}(t) S_{l}^{\alpha}\right\rangle_{T}$ is either periodic, if the two fundamental frequencies of the torus are commensurate, or quasiperiodic, if they are incommensurate. In either case, the intensity spectrum of $\left\langle S_{l}^{\alpha}(t) S_{l}^{\alpha}\right\rangle_{T}$ as determined by its Fourier transform 


$$
\Phi_{\alpha \alpha}^{(t)}(\omega)=\int_{-\infty}^{+\infty} d t e^{i c \omega t}\left\langle S_{l}^{\alpha}(t) S_{l}^{\alpha}\right)
$$

is discrete, governed by these two frequencies and, unless the time evolution of $S_{l}^{\alpha}(t)$ is harmonic, also by their sums and differences.

Quite generally, a continuous frequency spectrum is necessary to produce a correlation function which decays to a constant asymptotically for long times. In integrable twospin models, this can only be realized in autocorrelation functions of the type $\left\langle S_{i}^{\alpha}(t) S_{i}^{\alpha}\right\rangle_{p}$, which draw on the combined spectrum of the time evolution for all initial conditions. This spectrum is, in general, entirely continuous. For the purpose of illustration, we present here the exact autocorrelation functions $\left\langle S_{l}^{\alpha}(t) S_{l}^{\alpha}\right\rangle_{p}$ for two integrable cases of (1) for which the analysis is particularly simple.

(I) "Ising model" $\left(J_{x}=J_{y}=0, J_{z}=2, A_{x}=A_{y}=A_{z}\right.$ $=0)$ :

$$
\begin{aligned}
& \left\langle S_{l}^{x}(t) S_{l}^{x}\right\rangle_{P}=\frac{1}{3}[\sin (2 t) / 2 t], \\
& \Phi_{x x}^{(l)}(\omega)_{P}=(\pi / 6) \theta(2-|\omega|) .
\end{aligned}
$$

(II) Heisenberg nodel $\left(J_{x}=J_{y}=J_{z}=1, A_{x}=A_{y}\right.$ $=A_{z}=0$ ):

$$
\begin{aligned}
& \left\langle S_{l}^{x}(t) S_{l}^{x}\right\rangle_{p}=\frac{1}{6}+\frac{2}{3}\left(\frac{3}{4 t^{3}} \sin (2 t)\right. \\
& \left.-\frac{3+2 t^{2}}{8 t^{4}}+\frac{3-4 t^{2}}{8 t^{4}} \cos (2 t)\right) \text {, } \\
& \Phi_{x x}^{(l)}(\omega)_{p}=\frac{\pi}{3} \delta(\omega)+\frac{\pi}{24}|\omega|\left(4-\omega^{2}\right) \theta(2-|\omega|) .
\end{aligned}
$$

In both modeis, the correiation functions decay algebraical1y. The dominant term of the long-time asymptotic decay is determined by the strongest singularity in the continuous part of the intensity spectrum. In case (I), this is a discontinuity at $|\omega|=2$, which results in the $1 / t$ behavior of $(8 a)$. In case (II) the linear cusps at $\omega=0$ and $|\omega|=2$ give rise to the leading $1 / t^{2}$ terms in (9a). Note that in both models, the intensity spectrum has an upper frequency cutoff. This is so because the time evolution of $S_{l}^{a}(t)$ is harmonic for any initial conditions. This property is no longer present in the next simplest integrable case of the general two-spin model (1), the $X Y$ model $\left(J_{x}=J_{y}=1, J_{z}=0, A_{x}=A_{y}=A_{z}=0\right)$. Here the time evolution is expressible in terms of Jacobi elliptic functions for arbitrary initial conditions, ${ }^{5}$ and the intensity spectrum of the autocorrelation functions has no upper frequency bound.

In the nonintegrable cases of the two-spin model (1), i.e., for parameter values which do not satisfy the condition (4), oniy a subset of the phase-space trajectories is confined to invariant tori. For these regular trajectories, the autocorrelation functions $\left\langle S_{l}^{\alpha}(t) S_{l}^{\alpha}\right\rangle_{T}$ have the same characteristic properties as in the integrable cases discussed previously: no decay, discrete spectrum, two fundamental frequencies. However, in nonintegrable cases, the set of invariant tori is nowhere dense in phase space, even though it has a nonzero measure. Between any two tori, there is a region in which the phase flow is chaotic. Quite different characteristic features are expected of autocorrelation functions defined as time averages over chaotic trajectories.

For a specific example, we consider the nonintegrable case $J_{x}=J_{y}=1, J_{z}=0, A_{x}=2, A_{y}=-1, A_{z}=0$ of the two-spin model (1). We have evaluated for a particular set of initial conditions the three autocorrelation functions $\left\langle S_{1}^{\alpha}(t) S_{1}^{\alpha}\right\rangle_{T}, \alpha=x, y, z$, via the average over a time interval of length $T=100000$ from the time series of a chaotic trajectory. Figure 1 shows the function $\left\langle S_{1}^{x}(t) S_{1}^{x}\right\rangle_{T}$ for $0<t$ $<250$ and the functions $\left\langle S_{1}^{y}(t) S_{1}^{y}\right\rangle_{T},\left\langle S_{1}^{z}(t) S_{1}^{z}\right\rangle_{T}$ for time differences up to $t=100$. All three correlation functions clearly decay to zero. The rate of decay is slowest in $\left\langle S_{1}^{x}(t) S_{1}^{x}\right\rangle_{T}$ and fastest in $\left\langle S_{1}^{z}(t) S_{i}^{z}\right\rangle_{r}$. The $y y$ - and $z z^{-}$ correlation functions exhibit distinct oscillations unlike the $x x$-correlation function, which decays much more uniformly. The decay of these correlation functions results as a conksequence of the chaotic nature of the trajectory and implies that the phase flow in the region sampled by the trajectory has mixing character. The oscillations indicate that this mixing flow is superimposed on a quasiperiodic flow which is mostly perpendicular to the $x_{1}$ direction. They are signs of quasiperiodicity over short times, i.e., periodicity subject to continued phase slips. The inset to Fig. 1(a) shows $\left\langle S_{1}^{x}(t) S_{1}^{x}\right\rangle_{7}$ on a $\log -\log$ scale. For $20<t<100$, the curve is
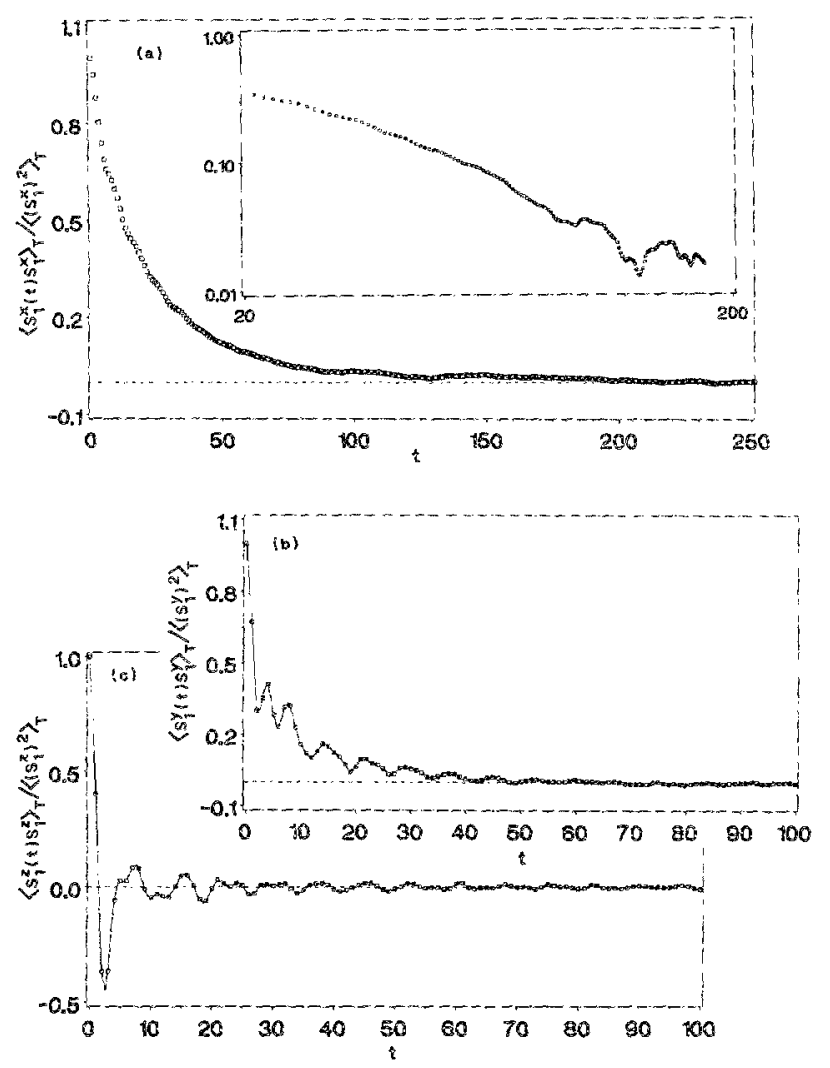

FIG. 1. Normalized awtocorreiation function $\left\langle S_{!}^{\alpha}(t) S_{1}^{\alpha}\right\rangle_{T} /\left\langle\left(S_{1}^{\alpha}\right)^{2}\right\rangle_{T}$, $a=x, y, z$, for the nonintegrable classical two-spin model defined by Hamiltonian (1) with parameter values $J_{x}=J_{y}=1, J_{z}=0, A_{x}=2, A_{y}=-1$, $A_{z}=0$. The results are obtained from the chaotic trajectory specified by the initial conditions $\vartheta_{i}^{(0)}=1.0, \vartheta_{2}^{(0)}=2.0, \varphi_{!}^{(0)}=3.0, \varphi_{2}^{(0)}=1.29$, and evalaated by a fourth-order Runge-Kutta integration via the time average over an interval of size $T=100000$. The inset to (a) shows the same correlation function $\left.\left\langle S_{1}^{x}\{t) S_{1}^{x}\right\rangle_{T} /\left(S_{1}^{x}\right)^{2}\right\rangle_{T}$ in a coubly logarithmic plot for tiznes between $t=20$ and $t=200$. 
nearly straight, indicating that the $x x$ correlations decay algebraically: $\left\langle S_{1}^{x}(t) S_{1}^{x}\right\rangle_{T} \sim t^{-1.5}$.

It is interesting to compare our findings with existing results for autocorrelation functions of area-preserving maps. A numerical study of the standard map ${ }^{6}$ yielded algebraic decay close to $\sim t^{-1 / 3}$; a similar study of the quadratic map $^{7}$ also found algebraic decay, but the exponent value could be placed only somewhere between $1 / 2$ and $3 / 2$. On the basis of a Markov chain model for transport in the boundary layer of a chaotic region, Manson, Cary, and Meiss ${ }^{8}$ predicted more rapic algebraic decay, $\sim t^{-2.05}$; a considerably slower algebraic decay, $\sim t^{-0.96}$, was predicted by the more elaborate calculation of Meiss and Ott $^{9}$ based on a Markov tree model. We can place our result among these others as discussed in the following.

In a previous comprehensive study ${ }^{5}$ of time averages in classical two-spin systems, we have already found evidence that the phase flow in chaotic regions is ergodic, but also that the convergence of time averages can be extremely slow due to long-time anomailes caused by low-fux cantori via two different mechanisms: (i) In a region of widespread chaos, the dominant mechanism is attributabie to the stickiness of its boundaries. They typically consist of layers of cantori separated by Birkhof chains, in which chaotic trajectories become temporarily trapped. We have found that the slowing down of convergence due to this mechanism is considerable but not alarming. (ii) A different mechanism, which is responsible for much stronger long-time anomalies, occurs in situations where a chaotic region is tessellated by cantori into roughly equal-sized compartnents. A characteristic feature of this case is that time averages appear to converge slowly to some mean value, similar to case (i), but then suddenly start to converge toward a different value [again slowly as in case (i)], and that this abrupt change happens repeatedly but irregularly, each change occurring as the trajectory switches compartments through one of the low-flux cantori. The time scales of this process are typically much larger than in process (i). The averages over the trajectory at hand have converged quite well. Thus the series must be long compared to any anomalies encountered, and the time scale for the remaining anomalies to be met must be very long compared to the averaging time used here.

Evidently, these long-time anomalies must play an im-

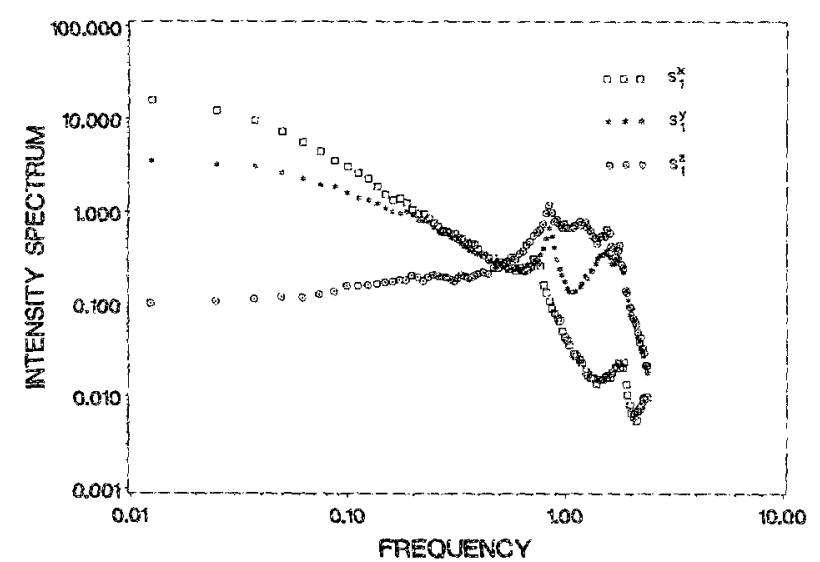

FIG. 2. Intensity spectra of the correlation functions $\left\langle S_{1}^{\alpha}(t) S_{i}^{\alpha}\right\rangle_{T}$, $\alpha=x, y, z$, shown in Fig. 1, as determined directly from the original time series $S_{1}^{\alpha}(t)$ of the chaotic trajectory. portant role in dynamic correlation functions. In $K$ systems, which are characterized by a special type of mixing fow (a phase flow associated with a positive Kolmogorov entropy production , and where none of the long-time anomalies described above can occur, the autocorrelation functions decay exponentially in time. ${ }^{10}$ In the model of Ref. 8 , the effect of only one sticky boundary is considered, and fast algebraic decay ensues. In Ref. 9 the effects of a fractal structure of sticky boundaries are included, which results in considerably slower algebraic decay. Since we are, as described previously, in an intermediate region, the intermediate exponent value is to be expected.

We have also determined the intensity spectra of $\left\langle S_{1}^{a x}(t) S_{1}^{\alpha}\right\rangle_{T}, \alpha=x, y, z$, directly from the time series of the chaotic trajectory (see Fig. 2). Each spectrum is continuous, as is characteristic of a chaotic trajectory. We observe marked enhancements at $\omega \approx 0.8$ and $\omega \approx 1.3$ in the spectra of $S_{1}^{y}$ and $S_{1}^{z}$. These oscillations are, as noted above, apparent in the correlation functions $\left\langle S_{1}^{y}(t) S_{1}\right\rangle_{T}$ and $\left\langle S_{1}^{z}(t) S_{1}^{z}\right\rangle_{T}$ (see Fig. 1). Our results also indicate that none of the spectra diverges in the low-frequency limit $\omega \rightarrow 0$, which is consistent with the rapid algebraic decay of the correlation functions.

In summary, we have found that algebraic decay of autocorrelation functions occurs in nonintegrable two-spin systems already for time averages over single (chaotic) trajectories. In integrable two-spin models, by contrast, autocorrelation functions decay only as a result of phase averag. ing.

A number of interesting questions remain to be answered: What is the role of invariant tor in the correlation functions $\left\langle S_{l}^{\alpha}(t) S_{l}^{\alpha}\right\rangle_{p}$ in nonintegrable models, given that the tori are nowhere dense in phase space? How do the effects of nonintegrability identified here for classical spin clusters make their appearance in autocorrelation functions of quantum spin systems near the classical limit? A more systematic study of dynamic correlation functions in integrable and nonintegrable classical and quantum spin clusters, which attempts to answer these and other questions, is currently in progress.

We thank F. Thomas and $\mathrm{R}$. Weber for their continued interest in this project. This work was supported by a grant from Research Corporation, by the U. S. National Science Foundation, Grant No. DMR-86-03036 and by Sigma Xi, the Scientific Research Society.

${ }^{1}$ M. Feingold, N. Moiseyev, and A. Peres, Phys. Rev. A 30, 509 (1984); H. Frahm and H. J. Mikeska, Z. Phys. B 60, 117 (1985); K. Nakamura and A. R. Bishop, Phys. Rev. B 33, 1963 (1986).

${ }^{2}$ G. Müller, Phys. Rev. A 34, 3345 (1986).

${ }^{3} \mathrm{E}$. Magyari, H. Thomas, R. Weber, C. Kaufman, and G. Müller, Z. Phys. B 65, 363 (1987).

${ }^{4}$ N. Srivastaya, C. Kaufman, G. Müller, E. Magyari, R. Weber, and H. Thomas, J. Appl. Phys. 61, 4438 (1987).

${ }^{5}$ N. Srivastava, C. Kaufman, G. Müller, R. Weber, and H. Thomas, Z. Phys. $B$ (in press).

${ }^{\circ}$ B. V. Chirikov and D. L. Shepalyansky, Physica 13R, 395 (1984).

'C. F. F. Karney, Physica 8D, 360 (1983).

8. D. Manson, J. R. Cary, and J. D. Meiss, J. Stat. Phys. 39, 327 (1985).

${ }^{9}$ I. D. Meiss and E. Ott, Phys. Rev. Lett. 55, 2741 (1985); Physica 20D, 387 (1986).

${ }^{10} \mathrm{G}$. M. Zaslavsky, Choos in Dynamic Systems (Harwood, New York, 1985). 